Social networks como estrategia Brand Engagement del sector comercial de la provincia de Tungurahua

\title{
Social networks como estrategia Brand Engagement del sector comercial de la provincia de Tungurahua
}

\section{Social networks como estrategia Brand Engagement del sector comercial de la provincia de Tungurahua}

\section{Redes sociais como Marca Estratégica Envolvimento do setor comercial da província de Tungurahua}

\author{
Wilson Ibarra-León ${ }^{\mathrm{I}}$ \\ wilson.ibarrale@gmail.com \\ Jesenia Escalante-Ibarra ${ }^{\text {II }}$ \\ jesenia-esca@hotmail.com
}

Leonardo Ballesteros-López ${ }^{\text {III }}$

leonardo.ballester@gmail.com

\author{
César Guerrero-Velasteguí IV \\ cesar-velastegu@gmail.com
}

Recibido: 27 de junio de 2018 * Corregido: 28 de agosto de 2018 * Aceptado: 20 de septiembre de 2018

Este artículo se enmarca en los resultados previos del proyecto de investigación institucional: "INFLUENCIA DE LA PUBLICIDAD EN EL COMPORTAMIENTO DEL CONSUMIDOR DEL SECTOR COMERCIAL DE LA PROVINCIA DE TUNGURAHUA", presentado en la Dirección de Investigación y Desarrollo DIDE, de la Universidad Técnica de Ambato y aprobado según resolución de Consejo Universitario: 2386-CU-P2017, del 15 de noviembre de 2017; proyecto coordinado por Ing. MBA. Leonardo Ballesteros López. 


\section{Resumen}

Conforme la administración del marketing ha evolucionado, durante las últimas décadas, sus objetivos y estrategias han cambiado, de un énfasis en ventas y participación (atraer clientes) a ganancias y fidelidad (compromiso con la marca). El estudio se enmarca en identificar los factores que inciden en el manejo de las redes sociales para el mejoramiento del Brand engagement en la provincia de Tungurahua. La investigación presenta un enfoque cualitativo y alcance correlacional. La muestra proviene de 384 consumidores. Se comprueba la hipótesis de estudio, las redes sociales influyen positivamente en la estrategia de brand engagement. Los resultados principales muestran que los contenidos que atraen mayor atención de usuarios en las redes sociales son: noticias y novedades, comentarios, concursos y sorteos de productos y promociones. Se concluye que, los consumidores innovadores no solo están interesados en cosas nuevas, sino que también están interesados en representarse a sí mismos a través de marcas asociadas con nuevos productos.

Palabras claves: Brand engagement; Compromiso de Marca; Intención de compra; Social networks. 


\begin{abstract}
As the marketing administration has evolved, during the last decades, its objectives and strategies have changed, from an emphasis on sales and participation (attracting customers) to profits and loyalty (commitment to the brand). The study is framed in identifying the factors that influence the management of social networks for the improvement of Brand engagement in the province of Tungurahua. The research presents a qualitative approach and correlational scope. The sample comes from 384 consumers. The study hypothesis is checked, social networks have a positive influence on the brand engagement strategy. The main results show that the contents that attract the most attention of users in social networks are: news and news, comments, contests and product and promotions sweepstakes. It concludes that innovative consumers are not only interested in new things, but are also interested in representing themselves through brands associated with new products.
\end{abstract}

Key words: Brand engagement; Brand Commitment; Intent to purchase; Social networks. 


\section{Introducción.}

A medida que la dirección del marketing o mercadeo ha evolucionado, durante las últimas décadas, los objetivos de las estrategias de marketing han cambiado de un énfasis en ventas y participación (atraer clientes) a ganancias y lealtad (retener clientes) (García y Sánchez, 2014; Segarra, Hidalgo, y Tur, 2016). Actualmente, la teoría de la dirección de marketing está cambiando nuevamente la forma en que se expresan sus objetivos para enfatizar el establecimiento de "relaciones" con los clientes que subyacen en el valor a largo plazo de cada cliente. Este último movimiento en la evolución del marketing, ha estimulado en parte por el desarrollo de la teoría y práctica de la gestión de la relación con el cliente (Segarra y otros, 2016). Además del concepto cambiante de "producto" sugerido por la lógica dominante del marketing de servicios.

El desconocimiento o desconfianza de una adecuada presencia en los social networks por parte de los empresarios, microempresarios y propietarios de negocios, ha generado un lento desarrollo en sus actividades comerciales con relación a su entorno digital y una mínima inversión publicitaria en este rubro (Masaquiza, Ballesteros, y González, 2017). Con el propósito de obtener clientes de por vida, se sugiere a los mercadólogos que colaboren y experimenten con ellos para que cada cliente se manifieste, a sí mismo, como socio de la empresa, puesto que, de esta manera se satisfará sus deseos y necesidades de forma altamente personalizada.

Investigaciones anteriores llegan a una reflexión en común con este problema y recomiendan qué para alcanzar las relaciones deseadas, las empresas deben alentar a los clientes a interactuar y participar con sus marcas (de Aguilera-Moyano, Baños-González, y Ramírez-Perdiguero, 2015). El compromiso con las marcas produce un alto uso, lealtad y un boca a boca positivo. Por lo tanto, el concepto de Brand engagement o compromiso de marca se ha convertido en un objetivo clave de

\footnotetext{
121 Vol. 5, núm. 1, enero 2019, pp. 118-135 Wilson Ibarra-León, Jesenia Escalante-Ibarra, Leonardo Ballesteros-López, César GuerreroVelasteguí
} 
varias estrategias de marketing (Cuillierier, 2016). Al incitar a los consumidores ir más allá del consumo funcional de productos para resolver sus problemas, las estrategias de compromiso de marca alientan a los consumidores a utilizar marcas para expresar sus autoconceptos (Wong y Merrilees, 2014). Cuando la marca empieza a formar parte íntimamente de la vida de un consumidor, esta adquiere un valor e importancia que va más allá del alcance habitual del significado de la misma, a manera de consolidar la relación y alcanzar el objetivo deseado de una relación a largo plazo. Es así, que los cambios de tendencia del consumidor y los primeros compradores de nuevos productos, son decisivos para el éxito de muchos nuevos productos (Puelles, 2014). El objetivo del estudio es analizar los factores que influyen en los Social Networks en el mejoramiento del Brand Engagement.

En el Ecuador, se presenta una tendencia de repunte de usuarios en los social networks tales como Facebook, Twitter e Instagram desde hace cinco años atrás. Esta influencia tecnológica no es exclusiva de los medios tradicionales de comunicación o de grandes grupos empresariales, sino que esta globalización apertura nuevos mercados y ha permitido que pequeños y grandes tengan la opción de tener presencia publicitaria, de contenidos y de oportunidades de negocio; por lo tanto, se prevé demostrar la relación de los social networks y su influencia en la estrategia de brand engagement (García y Sánchez, 2014; Masaquiza y otros, 2017).

Esto ha permitido ampliar posibilidades comerciales en el sector, tomando en cuenta que estas se han vuelto parte de su estilo de vida y cotidianidad en todos los niveles socioeconómicos. Por lo tanto, cuanto más se logre aprender sobre ellos, los gerentes podrán diseñar nuevas estrategias eficaces, al momento de introducir nuevos productos para atraer a estos primeros compradores (Wong y Merrilees, 2014). Es probable que las empresas innovadoras tengan una alta participación en la marca, y así, hacer hincapié en cómo el nuevo producto puede expresar su autoconcepto y logre

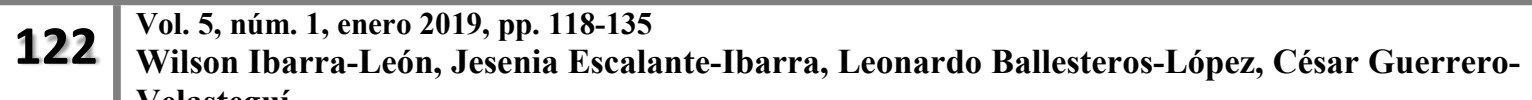
Velasteguí
} 
mejorar su aceptación. Se espera que a medida que se reúna más información sobre estos fenómenos, los profesionales de marketing estarán más capacitados para apelar a estas motivaciones de los consumidores (de Aguilera-Moyano y otros, 2015; Segarra y otros, 2016; Wong y Merrilees, 2014). Frente a esta problemática se establece la pregunta de investigación ¿Cómo influye la estrategia de social networks en el mejoramiento del Brand Engagement del sector comercial de la provincia de Tungurahua?

\section{Desarrollo.}

\section{Social networks}

En la actualidad, los social networks se presentan como la evolución de los medios de comunicación del ser humano, su avance se muestra a través del uso de nuevos canales de información. Su base se presenta en la creación, de conocimiento colectivo y confianza generalizada. Dentro de estos nuevos canales se logra hallar una multitud de clasificaciones diferentes como agregadores de noticias, wikis, blogs, entre otras, que usados en conjunto permiten potencializar la interacción con miles de personas con nuestras mismas inquietudes (Merrilees, 2016; Puelles, 2014). El propósito de los social networks es compartir información con nuestros amigos, y esta información puede ser texto, audio, video. Es evidente que nadie se adhiere a una red social porque necesita algún objeto, al contrario, quiere socializar con personas con sus mismos intereses, por lo que se deja claro que la gente no se encuentra en los social networks por temas comerciales (Gringarten, 2011).

Los social networks han creado nuevas maneras de comunicación en una comunidad digitalizada, pero si se relaciona con la comunicación, es la misma (Solem, 2015). De esta forma, para que una empresa tenga presencia en los medios digitales, debe ser gestionada con eficiencia, eficacia

\footnotetext{
123 Vol. 5, núm. 1, enero 2019, pp. 118-135 Wilson Ibarra-León, Jesenia Escalante-Ibarra, Leonardo Ballesteros-López, César GuerreroVelasteguí
} 
y efectividad (Gringarten, 2011; Masaquiza y otros, 2017; Solem, 2015). Para aquello, es indispensable contar con una estrategia claramente establecida que facilite seguir los lineamientos de una planificación. Es así, que, al momento de establecer la estrategia, la misma debe pasar por un proceso de cuestionamiento como: ¿Quieres comunicar tus contenidos a nuevos clientes?, ¿Te gustaría trabajar en red con personas y empresas afines?, ¿Quieras crear una comunidad de seguidores?, ¿Quieres hacer participar a tus clientes en el desarrollo de tu negocio?, ¿Quieres posicionarte como referente en tu sector?, ¿Te gustaría saber lo que se dice de tu empresa en Internet?

Hoy en día hay, existe una frase muy repetitiva por las personas involucradas en marketing digital: "Aunque no estés en redes sociales como empresa o como marca, realmente ya estás en ellas" (Kunerth y Mosley, 2011). Al encontrarse en un entorno, en el cual, las personas pueden hacer cualquier comentario en una conversación, en algún momento pleno, emerge como tema de discusión una determinada marca, ya sea bien o mal de ella. La discrepancia está en si las marcas realmente tienen intenciones de formar parte de una conversación y aclarar molestias o dudas de los consumidores (Kunerth y Mosley, 2011; Sarkar y Sreejesh, 2014). El fundador de Intuit destaca, "una marca ya no es lo que nosotros le decimos al consumidor que es. Ahora es lo que los consumidores dicen unos a otros que son”. Para Kabadayi y Price (2014) dirigir el mercado hacia los social networks requiere aprender una nueva manera de comunicarse con la audiencia en un ambiente digital, trata de desligarse del marketing tradicional, y hacer de ella nuevas prácticas, todo ello para poder comunicarse con el nuevo consumidor (Zakir, 2013). El esfuerzo que involucra estar presente en estos canales de comunicación digitales, hacen que las marcas presenten un cambio desde la raíz hacia el consumidor, puesto que, la opinión que se genere en estas plataformas debe ser tomada como un recurso valioso para la marca (Bylund y Lindgren, 2012). Es así, que esta estrategia digital, representa una serie de beneficios, como: conocer a su público objetivo, incrementa la capacidad de convocatoria

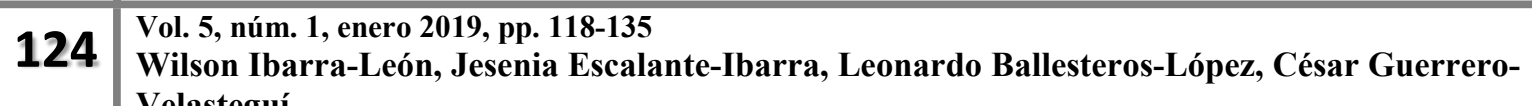
Velasteguí
} 
y de transmisión del mensaje de una empresa, disminuye los costos de comunicación, aporta transparencia de la marca, puesto que los consumidores aprueban o no, un producto con antelación (Mongolión, 2015).

\section{Innovación del consumidor}

De acuerdo a Hollebeek y Chen (2014) la innovación del consumidor se define como "una predisposición a comprar nuevos productos" (p. 2). El concepto ha sido investigado exhaustivamente para que varios escritos de comportamiento del consumidor detallen tanto el concepto como sus consecuencias (Hollebeek, 2011). La innovación del consumidor es considerada como un constructo específico de dominio, puesto que describe la predisposición a comprar nuevos productos en una categoría específica (L. Hollebeek, 2011b) o como una predisposición general hacia el mercado y categorías de productos (Alloza, 2008). Esta última orientación se adopta en el estudio actual para que coincida con el nivel en el que se conceptualiza el compromiso con la marca. La evidencia sugiere que la participación de la marca se asocia con la participación del producto y el conocimiento del producto (Goldsmith, Flynn, y Korzenny, 2015).

La hipótesis que se desarrolla es la comunicación en los social networks y el brand engagement están relacionados de manera positiva, puesto que otorga especial importancia a la participación y el conocimiento de las marcas por parte de ellas. Estas características son compartidas por los consumidores innovadores que están especialmente involucrados con su categoría de interés y saben mucho acerca de los productos y marcas en diferentes categorías (Cuillierier, 2016; Puelles, 2014)

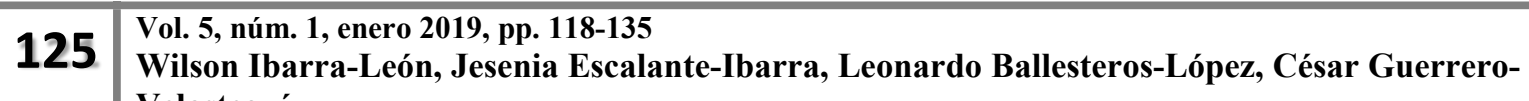
Velasteguí
} 


\section{Brand Engagement}

La amplia evidencia en literatura, apoya el argumento en el cual, los consumidores incorporan lo que poseen en su autoconcepto para lograr crear y expresar sus propios reflejos. Hace mucho tiempo, King, Varela, y Traávez (2012) argumentó que los productos suelen ser más que soluciones funcionales o utilitarias a los problemas; También, a la vez fungen como símbolos personales y sociales para los consumidores. Csikszentmihalyi y Rochberg-Halton (1981) hicieron uso de métodos etnográficos para describir cómo incluso los objetos más utilizados pueden generar un significado simbólico para los consumidores, incluso las cosas totalmente funcionales son utilizadas para socializar a un individuo a un cierto hábito o entorno de vida representativo. Varios psicólogos de consumo proponen que los consumidores puedan elegir y utilizar productos para expresar sus autoconcepto reales e ideales (Sprott, Czellar, y Spangenberg, 2009). Gringarten (2011) argumenta con fuerza sobre la base de una variedad de observaciones etnográficas que las posesiones eran un componente importante del sentido del yo, constituyendo un "yo extendido" que radica en el yo y la inclusión de mayores posesiones. La noción básica de que los productos son parte de uno mismo se puede hacer aún más específica cuando las marcas entran en escena. Las marcas tienen personalidad e imagen (Goldsmith y otros, 2015; Solem, 2016). Muchos académicos sustentan que el significado de las marcas para los consumidores es igual o más importante que los beneficios funcionales o utilitarios que brindan estas marcas. Puelles (2014) describen que los consumidores se relacionan con sus marcas por motivo de generar un significado psicológico a la misma. Hollebeek y Chen (2014) analiza en detalle el nuevo "espacio de consumo" donde los consumidores utilizan las marcas para construir sus realidades personales y sociales. Hollebeek (2011a) describe varios casos de marcas que se convierten en símbolos trascendentales para los consumidores, y varios casos en los cuales, el

\footnotetext{
126 Vol. 5, núm. 1, enero 2019, pp. 118-135
Wilson Ibarra-León, Jesenia Escalante-Ibarra, Leonardo Ballesteros-López, César Guerrero-
Velastegui Velasteguí
} 
consumidor crea un significado de marca independientemente de los gerentes de la marca (de Aguilera-Moyano y otros, 2015; Goldsmith y otros, 2015; Segarra y otros, 2016; Solem, 2016).

De esta manera, los mercadólogos proponen no solo que los consumidores formen relaciones con las marcas o se comprometan con ellas, sino que también difieren en la medida en que lo hacen y que esta variable de diferencia individual se puede medir de manera confiable. Parte de su esfuerzo de validación, consistió en demostrar que la participación de la marca se encuentra relacionada de manera positiva con el materialismo (Sprott y otros, 2009). A medida que crece el materialismo de las personas, buscan adquirir bienes materiales para compensar los bajos niveles de bienestar y salud psicológica (Sarkar y Sreejesh, 2014). Las personas materialistas a menudo tienen un frágil sentido de autoestima y forman malas relaciones con los demás (a quienes ven como objetos); por lo tanto, las marcas y la forma en que las marcas pueden representar el autoconcepto pueden llenar los vacíos en sus vidas, normalmente llenos de relaciones humanas. Merrilees (2016) también encontró que el brand engagement también se relacionó positivamente con vínculos más fuertes entre el autoconcepto y las marcas favoritas, un mejor recuerdo de los nombres de las posesiones actuales de la marca, prestando más atención a las marcas en la exposición incidental de la marca y la voluntad de esperar más tiempo para un nuevo Producto introducido por la marca favorita.

Este último hallazgo sugiere que los consumidores de alto nivel en consumismo buscan nuevos productos, al menos los de sus marcas favoritas (Hollebeek, 2011b; Puelles, 2014; Sarkar y Sreejesh, 2014; Sprott y otros, 2009). Por lo tanto, se cree que es importante examinar más a fondo este problema investigando si el compromiso de la marca está relacionado con la innovación del consumidor.

\footnotetext{
127 Vol. 5, núm. 1, enero 2019, pp. 118-135
Wilson Ibarra-León, Jesenia Escalante-Ibarra, Leonardo Ballesteros-López, César GuerreroVelasteguí
} 


\section{Materiales y Métodos}

El estudio emplea un enfoque cualitativo y alcance de investigación correlacional, como el concepto de Brand Engagement aparece en la literatura de marketing (Mongolión, 2015). La modalidad del estudio es bibliográfica y de campo, puesto que se utilizó la técnica de la encuesta, y como instrumento una serie de preguntas con respuestas cerradas que contribuirán a analizar los factores que influye en los social networks para el mejoramiento del Brand Engagement y para seleccionar los encuestados se utilizó la técnica de muestreo intencional. Los datos fueron recogidos de una muestra proveniente de los consumidores del sector comercial de la provincia de Tungurahua, se trabajó con 384 individuos (98 hombres y 286 mujeres). La información obtenida de las encuestas fue procesada en el software SPSS 25.0. El estadígrafo utilizado para corroborar la hipótesis planteada es el CHI-Cuadrado, puesto que contrasta de mejor manera la aseveración obtenida.

\section{Resultados}

El propósito del presente estudio fue evaluar la hipotética relación de los social networks y su influencia en la estrategia de brand engagement. A través del análisis de la encuesta se logró obtener los siguientes resultados:

\footnotetext{
128 Vol. 5, núm. 1, enero 2019, pp. 118-135 Wilson Ibarra-León, Jesenia Escalante-Ibarra, Leonardo Ballesteros-López, César GuerreroVelasteguí
} 
Dom. Cien., ISSN: 2477-8818

Vol. 5, núm.1. ene., 2019, pp. 118-135

Social networks como estrategia Brand Engagement del sector comercial de la provincia de Tungurahua

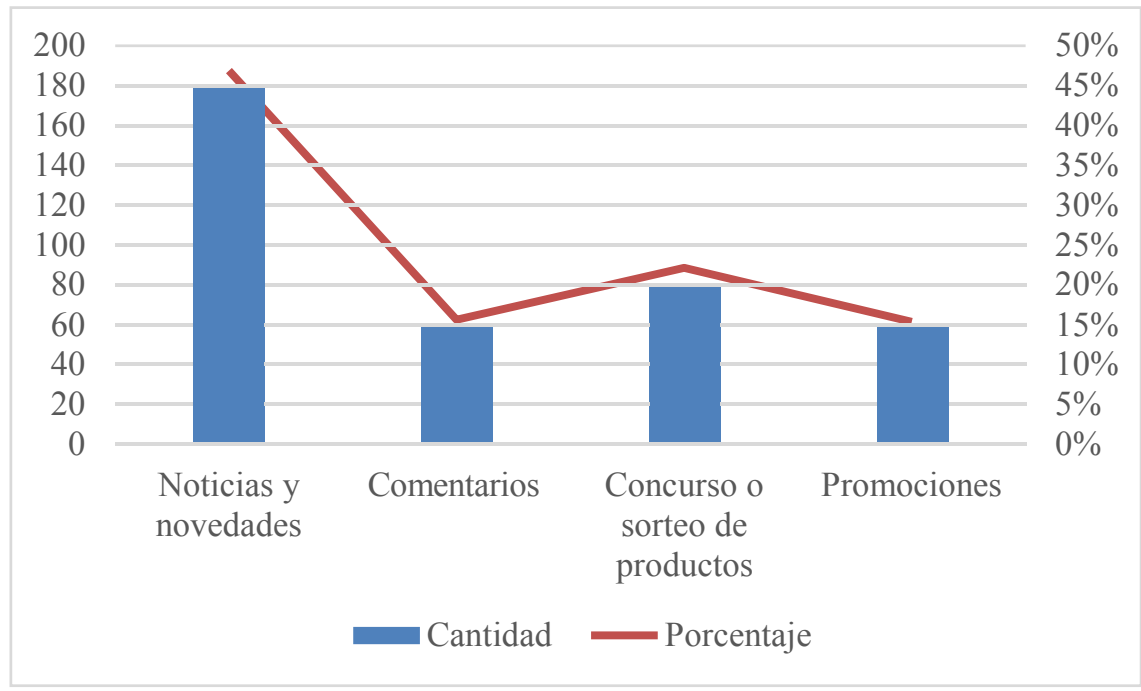

Gráfico 1. Qué tipo de contenidos llaman tu atención en las redes sociales

De acuerdo al gráfico 1. Los contenidos que llaman más la atención de los usuarios en las redes sociales son: noticias y novedades con un $47 \%$, comentarios $16 \%$, concursos y sorteos de productos con un $22 \%$ y promociones $15 \%$, lo que revela que las actividades más efectivas que debería emplear la marca en sus redes son: post de noticias y novedades, concursos y promociones.

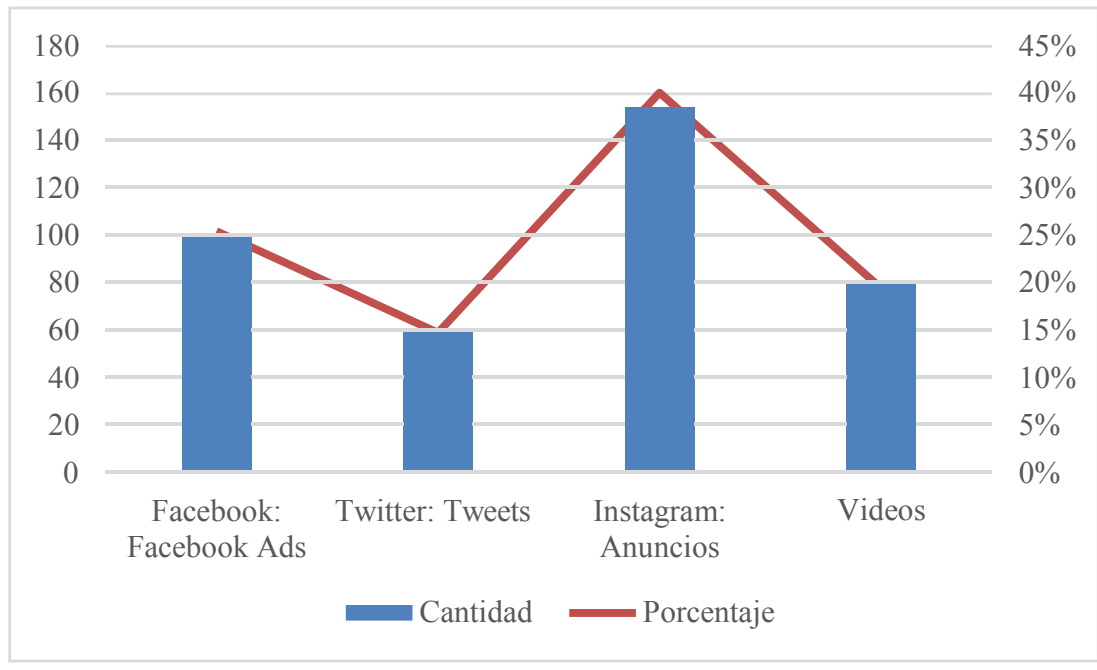

Gráfico 2. Qué formatos publicitarios le parecen más atractivos y captan su atención 
De acuerdo al gráfico 2. El 40\% de los encuestados indica que el formato publicitario que más ha observado en las redes sociales son los contenidos de Instagram, seguido de anuncios de Facebook Ads $26 \%$ y los videos con un $20 \%$ y finalmente Tweets y twitter con un $8 \%$.

De esta manera, se procede a correlacionar las preguntas detalladas anteriormente en los gráficos 1 y 2 como respuesta a la correlación de la hipótesis de debate. (tabla 1)

\section{Discusión}

Tabla 1. Pruebas de chi-cuadrado

\begin{tabular}{|c|c|c|c|}
\hline & Valor & gl & $\begin{array}{c}\text { Sig. asintótica } \\
\text { (bilateral) }\end{array}$ \\
\hline Chi-cuadrado de Pearson & $34,999^{\mathrm{a}}$ & 16 &, 004 \\
Razón de verosimilitudes & 39,611 & 16 &, 001 \\
Asociación lineal por lineal &, 928 & 1 &, 036 \\
N de casos válidos & 384 & & \\
\hline
\end{tabular}

a. 6 casillas $(24,0 \%)$ tienen una frecuencia esperada inferior a 5. La frecuencia mínima esperada es 1,21 .

Los resultados apoyaron la hipótesis. Como se observa en el Chi-cuadrado de Pearson, con relación a las dos variables de estudio, se ha obtenido un valor $\mathrm{p}$ de $(0,004)$; menor al valor de significancia $(0,05)$. Estos valores permiten aceptar la hipótesis de estudio, las redes sociales influyen positivamente en la estrategia de brand engagement. Por otro lado, se obtiene la razón de verosimilitudes, que permite identificar que si hay una similitud entre los valores esperados y observados. Asimismo, la verificación lineal por lineal establece un valor de $(0,036)$ lo que indica que los valores son auténticos y originales para el estudio. Por lo que se acepta la hipótesis alternativa y se rechaza la hipótesis nula, como lo demuestra el gráfico 3. 


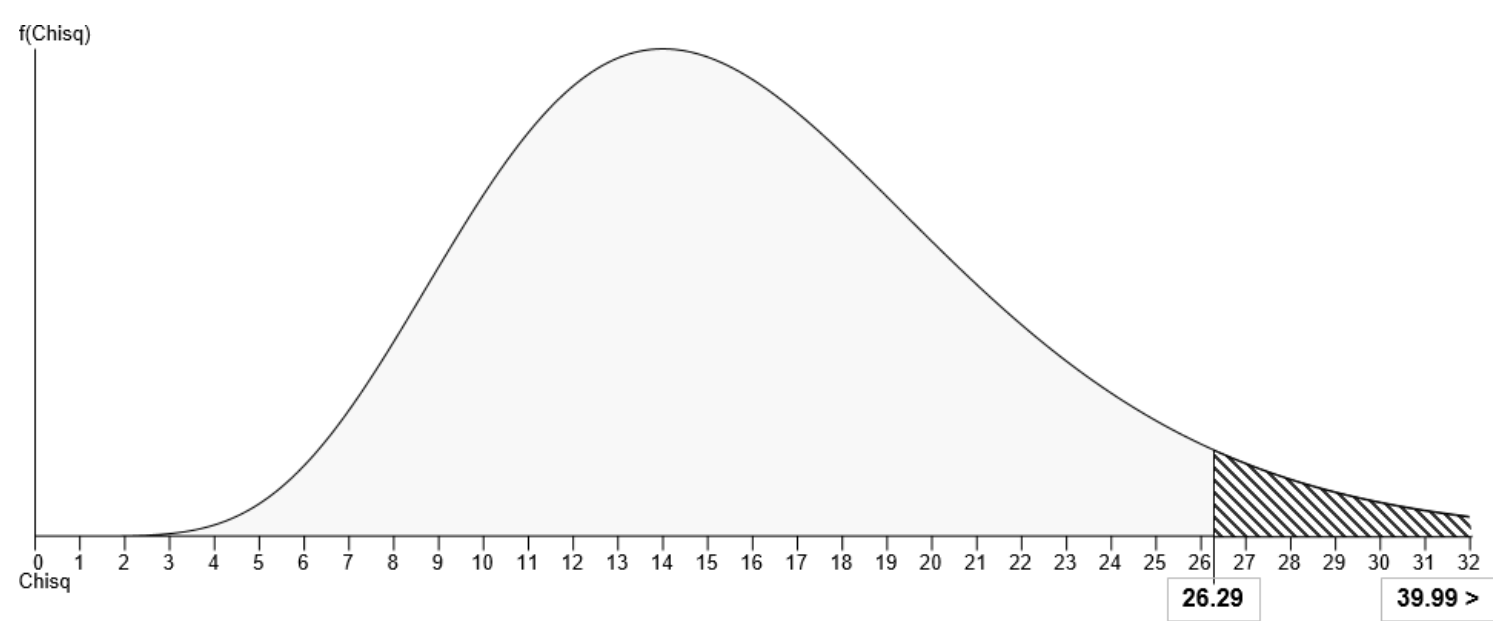

Gráfico 3. Campana de Gauss, correspondiente a la hipótesis de discusión.

Los consumidores que tienen un alto nivel de compromiso con la marca también parecen ser consumidores innovadores, lo que significa que están ansiosos por probar nuevos productos. Este hallazgo no solo se suma al extenso catálogo de información que tenemos sobre los consumidores innovadores, sino que también se comienza a ampliar lo que se conoce sobre el tema cada vez más importante del Brand Engagement. A medida que las empresas confían cada vez más en estrategias diseñadas para atraer a los consumidores con sus marcas, dicha información puede ser de gran utilidad para dar forma a estas estrategias. Aunque el estudio tiene sus limitaciones (la muestra no es representativa de la población total de Ecuador, las medidas son autoinformes y no de comportamiento, solo se utilizó una forma de método de encuesta). Por lo tanto, los hallazgos tienen implicaciones desde las perspectivas metodológicas, teóricas y gerenciales.

\section{Conclusiones.}

Actualmente, las PyMES de la provincia de Tungurahua no cuentan con perfiles personalizados en los Social Networks, esto deja al descubierto la inexistencia estandarizada en cuanto

\footnotetext{
131 Vol. 5, núm. 1, enero 2019, pp. 118-135

Wilson Ibarra-León, Jesenia Escalante-Ibarra, Leonardo Ballesteros-López, César GuerreroVelasteguí
} 
a imagen de marca, por lo cual, se debe tomar medidas correctivas necesarias con el fin de mejorar el branding visual de las marcas.

Los hallazgos tienen valor para la teoría del marketing. Primero, se tiene pruebas de que los consumidores innovadores no solo están interesados en cosas nuevas, sino que también están interesados en representarse a sí mismos a través de las marcas asociadas con los nuevos productos. Quizás estén mostrando o reforzando sus imágenes propias como personas que se embarcan temprano al comprar las marcas importantes. Otros estudios han encontrado que los consumidores innovadores tienen más probabilidades de ser buscadores de estatus (Gringarten, 2011; Kunerth y Mosley, 2011; Masaquiza y otros, 2017). Quizás su tendencia a usar nombres de marca como símbolos de autodenominación esté relacionado con el deseo de alcanzar un estatus. En segundo lugar, este estudio ayuda a agregar niveles más altos de innovación generalizada a la imagen que es el compromiso de la marca con el autoconcepto.

El principal factor táctico y estratégico que se debe considerar para fidelizar a los consumidores que se encuentran en los social networks es a través de la aplicación de una estrategia de marketing digital. La misma se fundamenta en establecer objetivos alcanzables, los cuales deben estar alineados a las cuatro C (Comunidad, Contexto, Contenido y Conexión). Se puntualiza que establecer objetivos admite diseñar un plan estratégico de social networks para la marca y mediante ellos se definen las herramientas Social Media a utilizar que faciliten escuchar y establecer conversaciones con los clientes, por lo cual, las marcas deben crear contenidos interactivos que sitúen el contexto de las empresas ofertantes y permitan al consumidor interactuar con la información, para obtener contenidos relevantes que proporcionen conexiones estables entre un grupo objetivo, y así, crear una comunidad digital que se identifique con la marca.

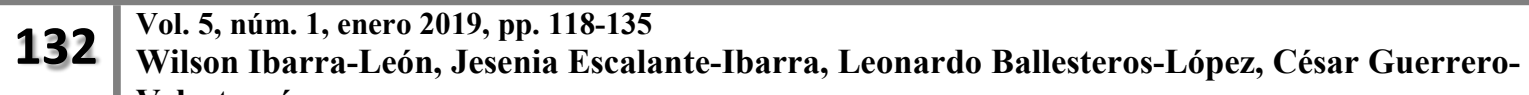
Velasteguí
} 
Finalmente, los gerentes pueden usar esta información de varias maneras como, darse cuenta de que los consumidores varían en la intensidad con la que se relacionan con las marcas y podría ser una clave valiosa para las estrategias de segmentación del mercado. Promover en gran medida las marcas a consumidores bajos sería una pérdida de tiempo y dinero, pero vincular cuidadosamente la imagen de marca con la autoimagen de los consumidores podría resultar muy valioso. Estos estilos de compra no se han vinculado hasta ahora con el compromiso con las marcas como se describe aquí.

Este nuevo hallazgo también proporciona información valiosa para los vendedores y minoristas. Por ejemplo, si se conocen los productos que los compradores impulsivos son propensos a comprar, a las marcas más rentables se les puede dar los lugares más destacados para que realicen compras impulsivas. Los compradores que "indecisos" merecen más estudio. Pueden ser cazadores de gangas que buscan ofertas o compradores recreativos que disfrutan de la experiencia. Los hallazgos de que los compradores basados en la necesidad y con descuento están menos comprometidos con la marca que los demás compradores encajan bien con los perfiles de los compradores frugales (Merrilees, 2016; Zakir, 2013) que probablemente estén más interesados en el precio que en las marcas. Los minoristas pueden usar algunas de las muchas herramientas disponibles para alentar el Brand engagement para cultivar los adjuntos de estos compradores para almacenar marcas cuyos precios más bajos los atraen.

\section{Futura línea de Investigación}

Los estudios futuros deben examinar las relaciones entre el Brand engagement y una variedad de importantes variables de diferencia individual del consumidor. Esta evidencia no solo mejoraría la comprensión del comportamiento del consumidor en el mercadólogo, sino que también, podría proporcionar a los gerentes información adicional sobre los rasgos de adquisición en los compradores

\footnotetext{
133 Vol. 5, núm. 1, enero 2019, pp. 118-135 Wilson Ibarra-León, Jesenia Escalante-Ibarra, Leonardo Ballesteros-López, César GuerreroVelasteguí
} 


\section{Referencias Bibliográficas.}

Alloza, A. (2008). Brand Engagement and Brand Experience at BBVA, the Transformation of a 150 Years Old Company. Corporate Reputation Review, 11(4), 371-379. https://doi.org/10.1057/crr.2008.31

Bylund, I., y Lindgren, S. (2012). Customer Engagement: A study of consumers interaction with fashion brands on social media Thesis. University of Borás.

Cuillierier, A. (2016). Customer engagement through social media. Helsinki Metropolia University of Applied Sciences. Helsinki Metropolia University of Applied Sciences. Recuperado a partir de https://www.theseus.fi/bitstream/handle/10024/115812/Thesis - Audrey Cuillierier - European and Business Administration.pdf?sequence $=1$

Csikszentmihalyi, M. y Rochberg-Halton, E. (1981), The Meaning of Things: Domestic Symbols and the Self. Cambridge, UK: Cambridge University Press.

de Aguilera-Moyano, J., Baños-González, M., y Ramírez-Perdiguero, J. (2015). Branded entertainment: Los contenidos de entretenimiento como herramienta de comunicación de marketing. Un estudio de su situación actual en España. Revista Latina de Comunicacion Social, 70(6), 519-538. https://doi.org/10.4185/RLCS-2015-1057

García, L., y Sánchez, R. (2014). Las Redes Sociales Como Herramienta De Mejora De La Experiencia Turística: Una Aplicación Al Sector Hotelero. Revista Iberoamericana de Turismo, 4(41), 16-34. Recuperado a partir de http://www.seer.ufal.br/index.php/ritur

Goldsmith, R. E., Flynn, L. R., y Korzenny, F. (2015). Brand Engagement and Consumer Innovativeness. Journal of Applied Marketing Theory, 6(1), 44-53.

Gringarten, H. (2011). Brand Engagement in Self-Concept: A Psychometric and Demographic Analysis. Journal of M ultidisciplinary, 3(3), 2-27.

Hollebeek, L. (2011a). Demystifying customer brand engagement: Exploring the loyalty nexus.
Journal
of Marketing
Management, 27(7-8),
785-807.

https://doi.org/10.1080/0267257X.2010.500132

Hollebeek, L. (2011b). Exploring customer brand engagement: Definition and themes. Journal of Strategic Marketing, 19(7), 555-573. https://doi.org/10.1080/0965254X.2011.599493

Hollebeek, L. D., y Chen, T. (2014). Exploring positively- versus negatively-valenced brand engagement: A conceptual model. Journal of Product and Brand Management, 23(1), 62-74. https://doi.org/10.1108/JPBM-06-2013-0332

Kabadayi, S., y Price, K. (2014). Consumer - Brand engagement on Facebook: liking and commenting behaviors. Journal of Research in Interactive Marketing, 8(3), 203-223. https://doi.org/10.1108/JRIM-12-2013-0081

King, K., Varela, M., y Traávez, C. (2012). Boletín mensual de análisis sectorial de MIPYMES. Tiungurahua. 
Kunerth, B., y Mosley, R. (2011). Applying employer brand management to employee engagement. Strategic HR Review, 10(3), 19-26. https://doi.org/10.1108/14754391111121874

Masaquiza, C., Ballesteros, L., y González, L. (2017). Análisis de los stakeholders como insumo de entrada en el diseño de un plan publicitario. Universidad, Ciencia y Tecnología, 1(Especial), 6067.

Merrilees, B. (2016). Interactive brand experience pathways to customer-brand engagement and value co-creation. Journal of Product and Brand Management, 25(5), 402-408. https://doi.org/10.1108/JPBM-04-2016-1151

Mongolión, P. (2015, junio). Gerenciamiento de comunicación y servicios de mercadeo. Ekos Negocios, 1-20.

Puelles, J. (2014). Fidelización de marca a través de redes sociales: Caso del Fan-page de Inca kola y el publico adolescente y joven, 241. https://doi.org/10.1097/PHM.0b013e3182412071

Sarkar, A., y Sreejesh, S. (2014). Examination of the roles played by brand love and jealousy in shaping customer engagement. Journal of Product and Brand Management, 23(1), 24-32. https://doi.org/10.1108/JPBM-05-2013-0315

Segarra, J., Hidalgo, T., y Tur, V. (2016). «Branded Webseries». Acciones Estratégicas Del Anunciante Basadas En La Ficción «Online» Corporativa Y El «Marketing» De Contenidos. Index. Comunicación, 6(2), 259-284.

Solem, B. A. A. (2015). The process of customer brand engagement in interactive contexts: Prerequisites, conceptual foundations, antecedents, and outcomes. Journal of applied microbiology.

Solem, B. A. A. (2016). Influences of customer participation and customer brand engagement on brand loyalty Service. Emerald Insight, 33(5), 332-342.

Sprott, D., Czellar, S., y Spangenberg, E. (2009). The Importance of a General Measure of Brand Engagement on Market Behavior: Development and Validation of a Scale. Journal of Marketing Research, 46(1), 92-104. https://doi.org/10.1509/jmkr.46.1.92

Wong, H. Y., y Merrilees, B. (2014). An empirical study of the antecedents and consequences of brand engagement. Marketing Intelligence $\{y\}$ Planning, 32(4), 413-435. https://doi.org/10.1108/02634501011078138

Zakir, M. (2013). Brand engagement: Insights and learning from other discipline. Mediterranean Journal of Social Sciences, 4(11), 609-614. https://doi.org/10.5901/mjss 2013.v4n11p609 